\title{
Control and Ultrafast Dynamics of a Two-Fluid Polariton Switch
}

\author{
M. De Giorgi, ${ }^{1,2, *}$ D. Ballarini, ${ }^{2}$ E. Cancellieri, ${ }^{3}$ F. M. Marchetti, ${ }^{3}$ M. H. Szymanska, ${ }^{4, \dagger}$ C. Tejedor, ${ }^{3}$ R. Cingolani, ${ }^{5}$ \\ E. Giacobino, ${ }^{6}$ A. Bramati, ${ }^{6}$ G. Gigli, ${ }^{1,2,7}$ and D. Sanvitto ${ }^{1,2}$ \\ ${ }^{1}$ NNL, Istituto Nanoscienze-CNR, Via Arnesano, 73100 Lecce, Italy \\ ${ }^{2}$ CBN, Istituto Italiano di Tecnologia, via Barsanti, 73010 Lecce, Italy \\ ${ }^{3}$ Departamento de Física Teórica de la Materia Condensada, Universidad Autónoma de Madrid, Madrid 28049, Spain \\ ${ }^{4}$ Department of Physics, University of Warwick, Coventry CV4 7AL, United Kingdom \\ ${ }^{5}$ Istituto Italiano di Tecnologia, IIT-Genova, Genova 16163, Italy \\ ${ }^{6}$ Laboratoire Kastler Brossel, Université Pierre et Marie Curie-Paris 6, École Normale Supérieure et CNRS, UPMC Case 74, \\ 4 place Jussieu, 75005 Paris, France \\ ${ }^{7}$ Innovation Engineering Department, University of Salento, Via Arnesano, 73100 Lecce, Italy
}

(Received 17 March 2012; published 27 December 2012)

\begin{abstract}
We investigate the cross interactions in a two-component polariton quantum fluid coherently driven by two independent pumping lasers tuned at different energies and momenta. We show that both the hysteresis cycles and the on-off threshold of one polariton signal can be entirely controlled by a second polariton fluid. Furthermore, we study the ultrafast switching dynamics of a driven polariton state, demonstrating the ability to control the polariton population with an external laser pulse, in less than a few picoseconds.
\end{abstract}

DOI: 10.1103/PhysRevLett.109.266407

PACS numbers: 71.36.+c, 42.65.Pc, 42.65.Sf

Although electronic technology has dominated a wide share of the market for communication and computation, becoming extremely sophisticated and well studied, it now seems unable to keep up with the same trend given the strong demand for fast networking performances and low energy consumption. Recent progress on photonic integrated circuits promises to overcome the limits of conventional electronic technology [1-5], offering the opportunity to realize efficient signal processing at speeds much higher than in conventional electronic devices, with further advantages for low energy consumption and negligible heating.

Optical switching is an essential component of optical communication networks and a fundamental milestone for quantum computation, allowing one optical signal to be controlled by another photonic beam. Various mechanisms have been proposed to achieve all-optical switching devices such as photonic-band-gap shift [6,7] and defectmode shift $[8,9]$. Here, the refractive-index contrast of the material is modified via a $\chi^{2}$ nonlinearity by an external optical pump, which, however, requires high intensities (often of the order of $\mathrm{GW} / \mathrm{cm}^{2}$ ) to achieve large switching efficiencies $[8,10,11]$. Another approach is to use materials with large nonlinear optical coefficients but with slower nonlinear response times [12]. Recently, a proof of principle demonstration of excitonic switching devices was reported [13]. The advantage of using excitons is their strong $\chi^{3}$ nonlinearities, with the drawback of being limited by their very slow intrinsic lifetime. On the other hand, microcavity polaritons, the strongly coupled quasiparticles between a quantum well exciton and a cavity photon, are especially interesting and promising systems to study, since they combine the properties of photons with the high nonlinearity of excitons. As bosonic quasiparticles, polaritons have unique coherent properties that have led to the achievement of Bose-Einstein condensation and superfluidity [14-16]. In addition, polariton-polariton interaction $[17,18]$ is at the basis of phenomena such as optical bistabilities [19,20], optical parametric oscillator [21], spin dependent activation of the bistable behavior [22,23], polariton switch operations via an additional nonresonant laser [24], and, more recently, transistor devices [25]. The polariton system is thus the ideal candidate for high-speed operations in logic devices at low energy consumption.

Optical bistability in microcavities is the enhanced resonant absorption of a detuned optical laser pump because of polariton interactions [26]: at low laser powers, by tuning the pump above the polariton dispersion, the driven polariton state is almost empty ("off" state). Increasing the power, the polariton blue-shift due to interactions causes enhanced absorption, a superlinear growth of the polariton population, and, eventually, a jump into a high polariton density ("on" state). The emission intensity versus the pump power is characterized by a hysteresis loop, which strongly depends on the excitation conditions [27]. In the case of two pumping lasers, however, the mutual interactions between the two driven polariton states, imply a richer phenomenology.

In this Letter, we investigate a two-component polariton system coherently driven by two different lasers with independently tunable frequencies, wavevectors and intensities. Stimulated by the theoretical analysis of Refs. [28,29], we demonstrate the possibility of controlling the hysteresis cycles of a polariton state via another state. Further, using a combination of continuous wave $(\mathrm{cw})$ and pulsed laser beams, we show the possibility of ultrafast control of a polariton state, and in particular the ability to switch a state not only "on" but also "off" within a timescale of few picoseconds. 
We use two spatially overlapping continuous wave Ti: sapphire lasers (P1 and $\mathrm{P} 2$ ) with different $k$ vectors and frequencies that resonantly excite two polariton states in the lower polariton branch (LPB) in a GaAs/AlAs microcavity [front (back) reflectors with 21 (24) pairs] containing three $\mathrm{In}_{0.04} \mathrm{Ga}_{0.96} \mathrm{As} / \mathrm{GaAs}$ quantum wells (the sample is kept at $10 \mathrm{~K}$ ). We choose the $k$ vectors and frequencies of both lasers to ensure optical bistability for each of the two states independently (upper panel in Fig. 1). Momentum space images of the emission intensity from the two states $\mathrm{S} 1$ and $\mathrm{S} 2$ at the pump energy $E_{1}$ and $E_{2}$ and momentum of $k_{1}$ and $k_{2}$, respectively, were detected in transmission geometry by using a high-resolution imaging CCD camera coupled to a spectrometer.

At the same time, we theoretically simulate the experimental system by means of a generalized Gross-Pitaevskii equation for the cavity $\Psi_{C}$ and exciton $\Psi_{X}$ fields $(\hbar=1)$ :

$$
\begin{aligned}
i \partial_{t}\left(\begin{array}{l}
\psi_{X} \\
\psi_{C}
\end{array}\right) & =\left(\begin{array}{l}
0 \\
F
\end{array}\right)+\hat{H}\left(\begin{array}{c}
\psi_{X} \\
\psi_{C}
\end{array}\right) \\
\hat{H} & =\left(\begin{array}{cc}
\omega_{X}-i k_{X}+g_{X}\left|\psi_{X}\right|^{2} & \frac{\Omega_{R}}{2} \\
\frac{\Omega_{R}}{2} & \omega_{C}-\frac{\nabla^{2}}{2 m_{C}}-i k_{C}
\end{array}\right) .
\end{aligned}
$$

The fields are coupled by the Rabi splitting $\Omega_{R}$ and resonantly driven by the two external lasers $F=$ $f_{1}(r) e^{i\left(k_{1} r-E_{1} t\right)}+f_{2}(r) e^{i\left(k_{2} r-E_{2} t\right)}$ having frequencies and momenta close to the experimental ones $\left(E_{1}=-4.25 \mathrm{meV}\right.$, $E_{2}=-3.7 \mathrm{meV}$, measured with respect to $\omega_{X}, k_{1}=$ $\left.0.2 \mu \mathrm{m}^{-1}, k_{2}=0.3 \mu \mathrm{m}^{-1}\right)$. Here, $\kappa_{C}=0.1 \mathrm{meV}$ and $\kappa_{X}=0.001 \mathrm{meV}$ are the photon and exciton decay rates, $m_{C}=2 \times 10^{-5} m_{0}$ the photon mass $\left(m_{0}\right.$ is the electron mass), the exciton interaction strength $g_{X}$ is set to one by rescaling both fields $\Psi_{C, X}$ and pump intensities $f_{1,2}$. The method used to solve Eq. (1) is the same one employed in Ref. [28]; thus, we give here only a short account of it. We establish, within the linear response approximation and for homogeneous pumps $\left[f_{1,2}(r)=f_{1,2}\right]$, the stability of the system as a function of the two pump intensities. In other words, we first solve the Gross-Pitaevskii equation at the mean-field level, and then, we establish the dynamical stability of each solution to small fluctuations. In this regime we approximate the mean-field solutions of the system with $\Psi_{C, X}(\mathbf{r}, t)=\sum_{j=1,2} \Psi_{j C, X}^{s s} e^{i\left(\mathbf{k}_{j} \cdot \mathbf{r}-E_{j} t\right)}$, where $\left|\Psi^{s s}{ }_{j C, X}\right|^{2}$ are the photon and exciton populations at frequency $E_{j}$ and momentum $\mathbf{k}_{j}$.

For the system parameters specified above, we plot the stability phase diagram for the two pumped states as a function of the two rescaled pump powers $f_{1}^{\prime}$ and $f_{2}^{\prime}$ in Fig. 1. The color scheme means that the state S1 on the left panel and S2 on the right panel are weakly populated (off) in the yellow region; two solutions either weakly (off) or strongly (on) populated coexist in the two bistable regions in green; finally, in the red region only the strongly populated (on) solution is stable. It is interesting to note that the threshold values
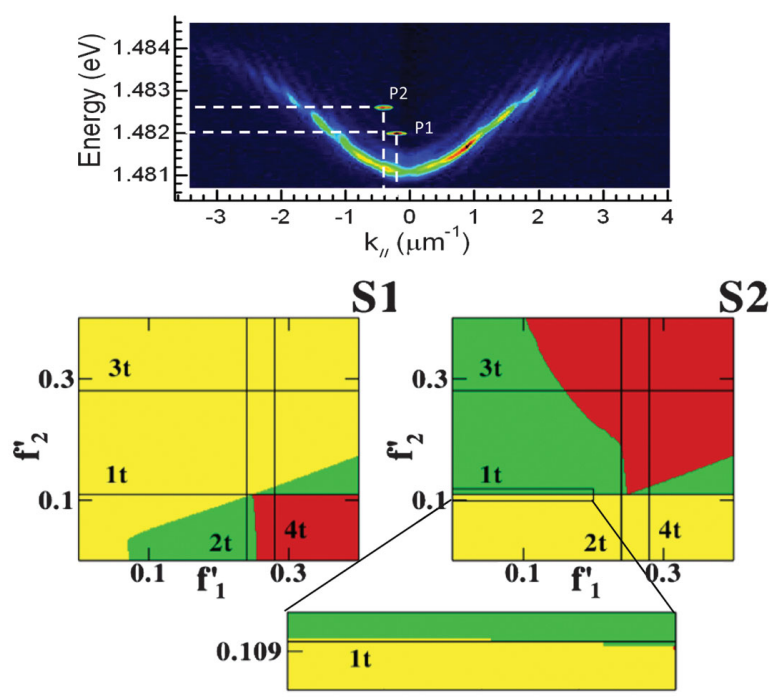

FIG. 1 (color). Upper panel: experimental polariton dispersion relation with superimposed two laser pumps at the corresponding energy $\left(E_{1}=1.482 \mathrm{eV}\right.$ and $\left.E_{2}=1.4826 \mathrm{eV}\right)$ and momenta $\left(k_{1}=0.2 \mu \mathrm{m}^{-1}\right.$ and $\left.k_{2}=0.4 \mu \mathrm{m}^{-1}\right)$ used to excite two different polariton states. Lower panels: theoretically evaluated phase diagram showing the off and on states of state S1 (left panel) and state S2 (right panel) as a function of the rescaled pump intensities $f_{1,2^{\prime}}=\sqrt{g_{X}} f_{1,2}\left[\mathrm{meV}^{3 / 2}\right]$. In the yellow region the states $\mathrm{S} 1$ and $\mathrm{S} 2$ can only be weakly populated (off), while in the red one they can only be strongly populated (on). In the green region both on and off solutions coexist (bistable region). The black thick vertical and horizontal lines correspond to the cases studied in Fig. 2, where one pump is kept constant while the other scans different intensity values.

between the on and the off states of one fluid can strongly depend on the intensity of the pump of the other state.

To better understand the behavior of the system, we compare, in Fig. 2, the experimental (1e, 2e, 3e, and 4e) and theoretical (1t, 2t, 3t, and 4t) emission intensities from the two states by either fixing the pump power P2 and changing P1 (left column of Fig. 2 and horizontal black lines in the phase diagram of Fig. 1), or by fixing P1 and changing P2 (right column of Fig. 2 and vertical black lines in the phase diagram of Fig. 1). We choose the values of the fixed pump powers to represent the most different configurations for the initial and final states. In both experimental and theoretical plots, the emission intensities are normalized to the maximum values of either the $\mathrm{S} 1$ or S2 state when they are excited independently.

In two cases (1 and 2) we start with the dressed LPB red detuned with respect to both lasers, while in the other two cases (3 and 4) we start with the LPB blue detuned with respect to laser 1 but red detuned with respect to laser 2. As can be observed from Fig. 2, by controlling the power of one pump, and therefore the population of the corresponding state (the hysteresis of which is shown with solid lines), we are able to control the population of the other polariton state (solid and open symbols for increasing and decreasing pump power, respectively). 

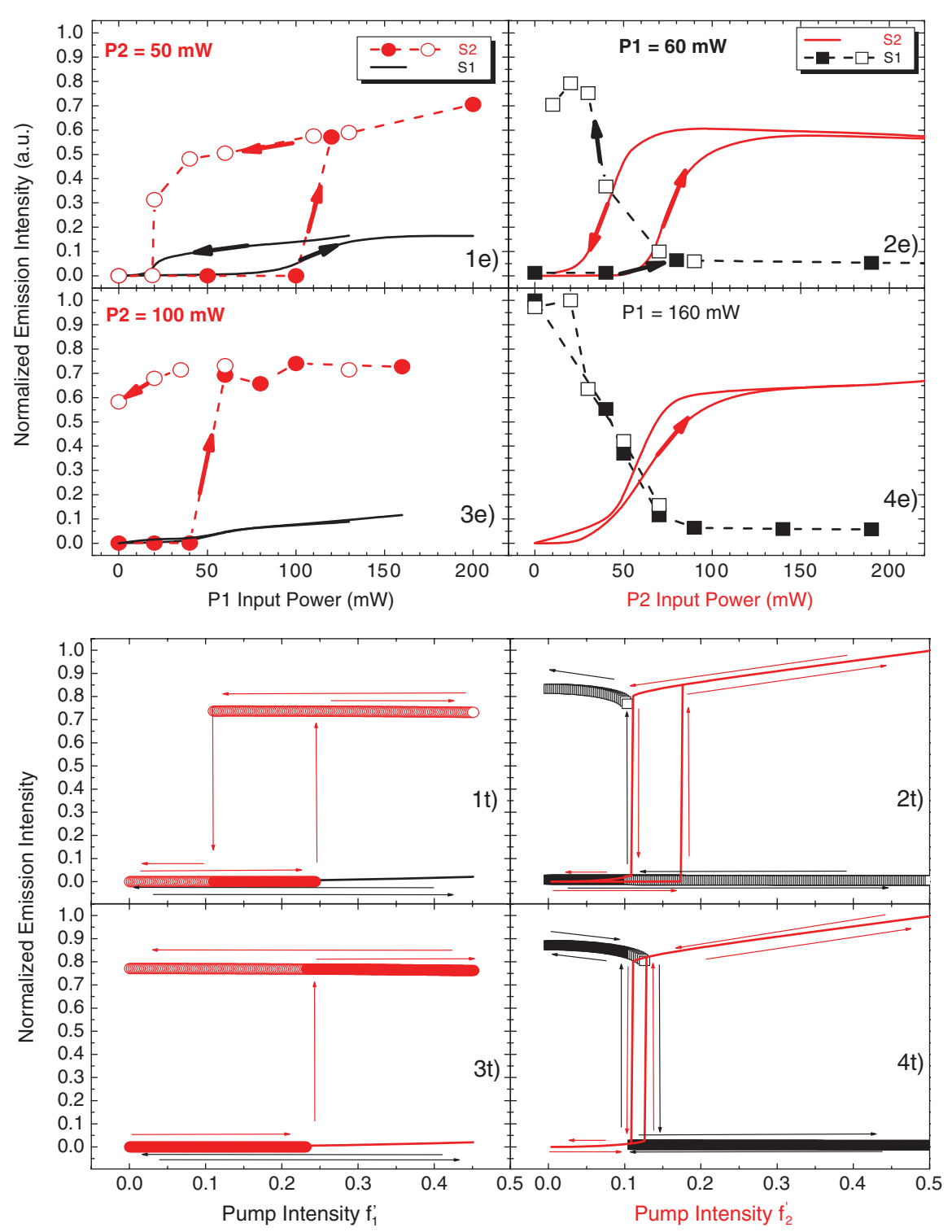

FIG. 2 (color). Experimental (1e, 2e, 3e, and 4e) and theoretical (1t, 2t, 3t, and 4t) normalized emission intensities from the two states $\mathrm{S} 1$ (black) and S2 (red) obtained by either changing the pump power P1 for fixed pump power P2 (left column), or changing pump power P2 for a fixed pump power P1 (right column). Arrows and symbols correspond to the power change variation: increasing power, solid symbol; decreasing power, open symbols. Note that for the theoretical emission intensity we consider $\left|\Psi^{S S}{ }_{1 C}\right|^{2}$ and $\left|\Psi^{S S}{ }_{2 C}\right|^{2}$, respectively, which are proportional to the experimental emission intensities when the Hopfield factors are taken into account.

In the first case (panels 1e and 1t), pump power P2 is fixed below the hysteresis cycle threshold of state $\mathrm{S} 2$ when the P1 pump power is 0 . Here, the LPB is red detuned with respect to both pumping lasers. By increasing the $\mathrm{P} 1 \mathrm{pump}$ power, the LPB becomes resonant with $E_{2}$ and is therefore pushed up far above $E_{1}$. In these conditions, the system is filled with polaritons in state S2 but with just a few polaritons in state $\mathrm{S} 1$, even when the P1 pump power reaches values above the threshold it would have when the P2 pump power is 0 . When the P1 pump power is again reduced to 0 , the intensity of the $\mathrm{P} 2$ pump power is not sufficient to keep the LPB blue detuned, and therefore the system goes back to the initial empty state. This demonstrates that a full hysteresis cycle of the state S2 can be completely controlled by the pump P1.

In the second case (panels $2 \mathrm{e}$ and $2 \mathrm{t}$ ), the initial conditions are similar to the previous case. Here, however, the $\mathrm{P} 2$ pump power is varied and the P1 pump power is kept constant to a value large enough to sustain the LPB in the on state of state $\mathrm{S} 1$. When pump power $\mathrm{P} 2$ is turned on, the LPB enters again in resonance with $E_{2}$ and is therefore far above $E_{1}$, and the cavity is filled with polaritons in state $\mathrm{S} 2$. In this case, however, when pump power $\mathrm{P} 2$ is decreased, even if the LPB red detunes to values smaller than $E_{2}$, it is sustained in resonance with $E_{1}$ by pump power P1, and therefore the cavity now remains filled with polaritons in 
state $\mathrm{S} 1$. The net effect of this cycle is that pump power P2 can be used to turn on state $\mathrm{S} 1$.

In the third case (panels $3 \mathrm{e}$ and $3 \mathrm{t}$ ), the LPB is blue detuned by the $\mathrm{P} 2$ pump power, so that it is higher than $E_{1}$ and lower than $E_{2}$. The value of pump power $\mathrm{P} 2$ is chosen in order to be strong enough to sustain the on state $\mathrm{S} 2$. When pump power P1 is increased, it pushes the LPB in resonance with $E_{2}$ and state $\mathrm{S} 2$ turns on while state $\mathrm{S} 1$ remains poorly populated. When pump power $\mathrm{P} 1$ is decreased to 0 , the LPB is sustained in resonance with $E_{2}$ by pump power $\mathrm{P} 2$. The net effect of this cycle is the opposite of the previous one, a cycle in the intensities of pump power $\mathrm{P} 1$ turns state $\mathrm{S} 2$ on.

Finally, in the fourth case (panels $4 \mathrm{e}$ and $4 \mathrm{t}$ ), the LPB is blue detuned to be higher than $E_{1}$ and lower than $E_{2}$. When pump power P2 is turned on, the LPB enters in resonance with $E_{2}$, it fills with polaritons in state $\mathrm{S} 2$, and it stays far above $E_{1}$, so that the population in state $\mathrm{S} 1$ decreases almost to 0 . When pump power P2 is turned off again, the LPB red detunes and goes back in resonance with $E_{1}$, and the system goes back to its initial conditions. As a consequence, the S1 state is reversibly switched on and off by turning off and on a different $\mathrm{S} 2$ state.

This allows one to control not only the on but also the off state of a polariton quantum fluid via another polariton state. From this analysis it emerges that, for two interacting polariton fluids, one polariton state can be used to control the population of the other state. Depending on both the relative intensities and the relative detuning of the two pumps, the system can be brought in and out of resonance with the pump frequencies, and so the two states can be turned on and off. As a final remark, we would like to address some differences between the theoretical curves and the experimental data in Fig. 2. While the theoretical curves present sharp transitions between the on and off states, as well as extremely low population in the off states, the experimental curves display smoother crossover and slightly populated off states. These differences can be in part ascribed to temperature fluctuations and time averaging over different realizations. Furthermore, one has to take into account that, while the theoretical analysis is carried on with $\delta$-like laser lines, in the experiments at least one of the two cw lasers was not a monomode laser, allowing for an effective broader excitation line, which justifies the observation of intermediate conditions. For the same reason, the bistable region of state S2 (see zoom in Fig. 1) depends on the pump power $f_{1}^{\prime}$ in a much weaker way in the theoretical analysis than in the experimental case.

To measure the on-off switching time of state S1, we pump the system in state $\mathrm{S} 2$ with a Ti:sapphire pulsed laser with a pulse width of $120 \mathrm{fs}$ and a repetition rate of $82 \mathrm{MHz}$, while state $\mathrm{S} 1$ is populated by the monomode cw laser with linewidth $<5 \mathrm{MHz}$. This has been performed on a different point of the sample with a more positive detuning, where the on-off switch effect is more pronounced. Energies and momenta of the two lasers are chosen as shown in the inset of Fig. 3(a)): because of the

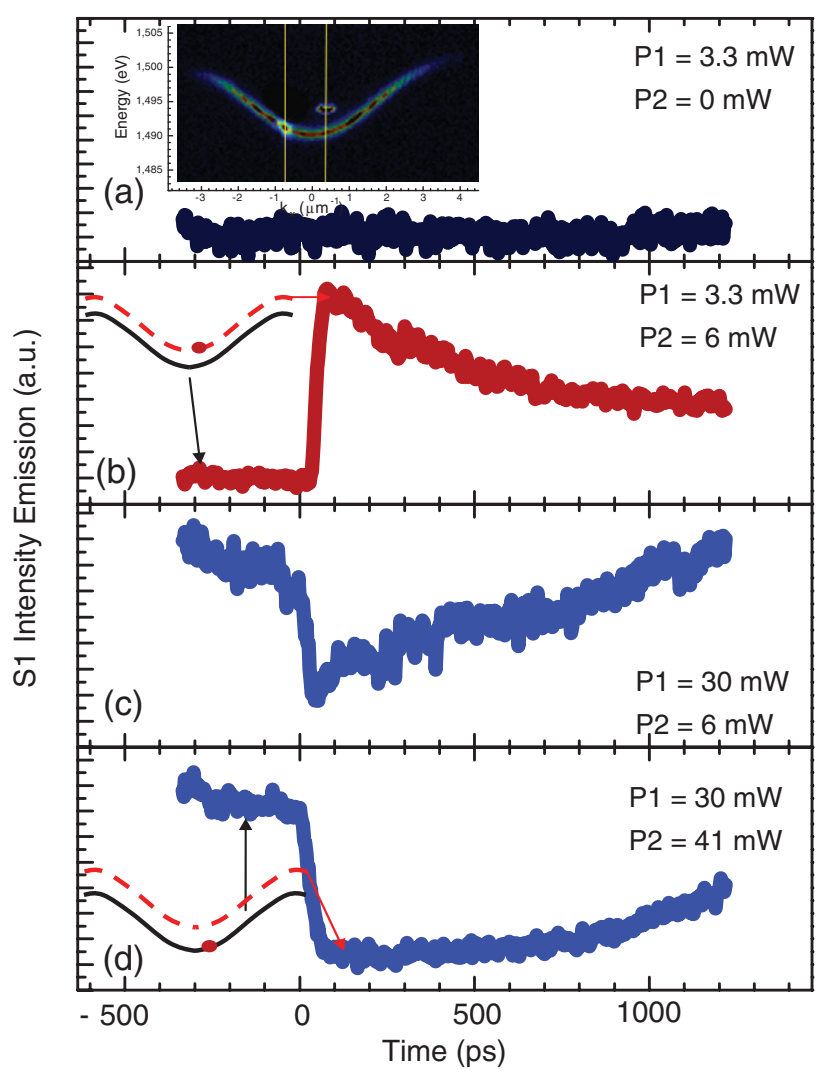

FIG. 3 (color). Time-resolved photoluminescence intensity of state $\mathrm{S} 1$ excited by a cw (P1) and a fs pulsed (P2) laser under the conditions shown in the inset of Fig. 2(a)): $k_{1}=0.35 \mu \mathrm{m}^{-1}$ and $E_{1}=1.494 \mathrm{eV}, k_{2}=0.73 \mu \mathrm{m}^{-1}$ and $E_{2}=1.491 \mathrm{eV}$. The spectra shown are obtained under four different excitation conditions: (a) the state is pumped only by the cw laser P1 at a very low pump power of $3.3 \mathrm{~mW}$. Under this condition state S1 is on an off state; (b) state $\mathrm{S} 1$, same as in (a) but a fs pulsed laser exciting the system with a low power P2 $=6 \mathrm{~mW}$ switches on the S1 state; (c), (d) state $\mathrm{S} 1$, in an on state (continuously pumped by $\mathrm{P} 1$ at $30 \mathrm{~mW}$ ), is switched off by the pulsed laser. The insets in (b) and (d) show the influence of a fs pulsed laser (P2) pumping the system resonantly. The continuous pump P1 is schematized by the red dots.

wide spectral range of the pulsed laser, pump power $\mathrm{P} 2$ is always on resonance with the LPB, whereas $E$ and $k$ of the $\mathrm{cw}$ laser pump power $\mathrm{P} 1$ has been chosen to avoid optical bistability for state $\mathrm{S} 1$ when the $\mathrm{P} 2$ pump power is 0 . Timeresolved photoluminescence of the $\mathrm{S} 1$ state is performed in transmission geometry by using a Hamamatsu streak camera coupled to a $0.55 \mathrm{~m}$ spectrometer (time resolution $5 \mathrm{ps}$ ).

The effect of the pulsed laser on state $\mathrm{S} 1$ is shown in Fig. 3 for different excitation conditions. Below threshold, when $E_{1}$ is still slightly detuned above the LPB energy, state $\mathrm{S} 1$ is off [black line in the inset of Fig. 3(b)]. The pulsed laser fast induces a blueshift [red dashed line in the inset of Fig. 3(b)], leading to a strong increase of the polariton population in state $\mathrm{S} 1$. This results in a switch on of the S1 state [Fig. 3(b)]. Once the pulse is gone away, the S1 state comes back to its original steady state, with a recovery time of hundreds of picoseconds. Conversely, 
above the pump P1 power threshold [Figs. 3(c) and 3(d)], when state $\mathrm{S} 1$ is already in the on state [black line in the inset of Fig. 3(d)], the further blueshift induced by the pulse laser (red dashed line) brings the S1 state out of resonance, resulting in a rapid reduction of the polariton population to less than one-half of its original density (off state). Similar to the previous case, the state S1 polariton population returns to the initial condition in many hundreds of picoseconds. This effect is further enhanced for higher power of the pulsed pump, resulting in a bigger variation of the polariton population between the on and off states [Fig. 3(d)], and in a longer recovery time. Such a long recovery time could be ascribed to a dynamical parametric instability, which is temporarily triggered by the ultrafast (and thus broad in energy) laser, similar to what has already been observed in recent TOPO experiments [30]. In all cases, we estimate switching on and off times for the $\mathrm{S} 1$ signal state of $5 \mathrm{ps}$, which is limited by the time resolution of the system (not visible in the long time range used for Fig. 3).

To conclude, we have studied the stability of a twocomponent polariton quantum fluid coherently driven by two independent pump lasers. We clearly demonstrate that we are able to control the hysteresis loop of a polariton quantum fluid by changing the population of a second polariton state and show how the polariton nonlinear optical properties can be used to switch not only on but also off a polariton fluid. Finally, we have observed a very short, of the order of a few picoseconds, switching time between the on and off states, establishing microcavity polaritons as promising systems for ultrafast optical operations.

The authors acknowledge P. Cazzato for the technical assistance with the experiments. This work has been partially funded by the FIRB Italnanonet, FIRB Italy-Japan HUB on nanotechnologies, the POLATOM ESF Research Networking Program, the Spanish MINECO (MAT201122997), CAM (S-2009/ESP-1503), and the program Ramon Y Cajal (F. M. M.).

*milena.degiorgi@unisalento.it

'Also at London Centre for Nanotechnology, London, United Kingdom.

[1] H. Volterra and M. Zimmerman, WDM Solutions 47 (2000).

[2] A. Daraei et al., Appl. Phys. Lett. 88, 051113 (2006).

[3] V.R. Almeida, C.A. Barrios, R.R. Panepucci, and M. Lipson, Nature (London) 431, 1081 (2004).

[4] R. Nagarajan et al., IEEE J. Sel. Top. Quantum Electron. 11, 50 (2005).

[5] R. Kirchain and L. C. Kimerling, Nat. Photonics 1, 303 (2007).

[6] M. Scalora, J.P. Dowling, C.M. Bowden, and M.J. Bloemer, Phys. Rev. Lett. 73, 1368 (1994).

[7] D. A. Mazurenko, R. Kerst, J. I. Dijkhuis, A. V. Akimov, V. G. Golubev, D. A. Kurdyukov, A.B. Pevtsov, and A. V. Sel'kin, Phys. Rev. Lett. 91, 213903 (2003).
[8] P. Barthelemy, M. Ghulinyan, Z. Gaburro, C. Toninelli, L. Pavesi, and D.S. Wiersma, Nat. Photonics 1, 172 (2007).

[9] S. Lan and H. J. Ishilawa, Appl. Phys. 91, 2573 (2002).

[10] M. Shimizu and T. Ishihara, Appl. Phys. Lett. 80, 2836 (2002).

[11] A. Hache and M. Bourgeois, Appl. Phys. Lett. 77, 4089 (2000).

[12] R. Katouf, T. Komikado, M. Itoh, T. Yatagai, and S. Umegaki, Photon. Nanostr. Fundam. Appl. 3, 116 (2005).

[13] G. Grosso, J. Graves, A. T. Hammack, A. A. High, L. V. Butov, M. Hanson, and A. C. Gossard, Nat. Photonics 3, 577 (2009).

[14] J. Kasprzak, M. Richard, S. Kundermann, A. Baas, P. Jeambrun, J. M. J. Keeling, F. M. Marchetti, M. H. Szymańska, R. André, J.L. Staehli, V. Savona, P. B. Littlewood, B. Deveaud, and L.S. Dang, Nature (London) 443, 409 (2006).

[15] A. Amo, D. Sanvitto, F. P. Laussy, D. Ballarini, E. del Valle, M.D. Martin, A. Lemaître, J. Bloch, D. N. Krizhanovskii, M. S. Skolnick, C. Tejedor, and L. Viña, Nature (London) 457, 291 (2009).

[16] A. Amo, J. Lefrère, S. Pigeon, C. Adrados, C. Ciuti, I. Carusotto, R. Houdré, E. Giacobino, and A. Bramati, Nat. Phys. 5, 805 (2009).

[17] P. G. Savvidis, J. J. Baumberg, R. M. Stevenson, M. S. Skolnick, D. M. Whittaker, and J.S. Roberts, Phys. Rev. Lett. 84, 1547 (2000).

[18] C. Diederichs, J. Tignon, G. Dasbach, C. Ciuti, A. Lemaître, J. Bloch, Ph. Roussignol, and C. Delalande, Nature (London) 440, 904 (2006).

[19] A. Baas, J.P. Karr, M. Romanelli, A. Bramati, and E. Giacobino, Phys. Rev. B 70, 161307(R) (2004).

[20] H. M. Gibbs, Optical Bistability: Controlling Light with Light (Academic Press, Orlando, FL, 1985).

[21] R. M. Stevenson, V. N. Astratov, M.S. Skolnick, D. M. Whittaker, M. Emam-Ismail, A. I. Tartakovskii, P. G. Savvidis, J. J. Baumberg, and J. S. Roberts, Phys. Rev. Lett. 85, 3680 (2000).

[22] A. Amo, T.C.H. Liew, C. Adrados, R. Houdré, E. Giacobino, A. V. Kavokin, and A. Bramati, Nat. Photonics 4, 361 (2010).

[23] K. Paraiso, M. Wouters, Y. Léger, F. Morier-Genoud, and B. Deveaud-Plédran, Nat. Mater. 9, 655 (2010).

[24] T. Gao, P. S. Eldridge, T.C.H. Liew, S.I. Tsintzos, G. Stavrinidis, G. Deligeorgis, Z. Hatzopoulos, and P. G. Savvidis, Phys. Rev. B 85, 235102 (2012).

[25] D. Ballarini et al., arXiv:1201.4071.

[26] V. D. Kulakovskii, A. I. Tartakovskii, D. N. Krizhanovskii, A. Armitage, J. S. Roberts, and M. S. Skolnick, Phys. Usp. 43, 853 (2000).

[27] D. M. Whittaker, Phys. Rev. B 71, 115301 (2005).

[28] E. Cancellieri, F. M. Marchetti, M. H. Szymanska, and C. Tejedor, Phys. Rev. B 83, 214507 (2011).

[29] E. Cancellieri, F. M. Marchetti, M. H. Szymańska, D. Sanvitto, and C. Tejedor, Phys. Rev. Lett. 108, 065301 (2012).

[30] D. Ballarini, D. Sanvitto, A. Amo, L. Viña, M. Wouters, I. Carusotto, A. Lemaitre, and J. Bloch, Phys. Rev. Lett. 102, 056402 (2009). 